\title{
Immunotherapy in lung cancer: the chemotherapy conundrum
}

\author{
Laxmi Narayana R. Buddharaju ${ }^{1}$, Apar Kishor Ganti ${ }^{1,2}$ \\ ${ }^{1}$ Division of Oncology-Hematology, Department of Internal Medicine, University of Nebraska Medical Center, Omaha, NE, USA; ${ }^{2}$ Division of \\ Oncology-Hematology, Department of Internal Medicine, VA-Nebraska, Western Iowa Health Care System, Omaha, NE, USA \\ Correspondence to: Apar Kishor Ganti, MD, MS. Division of Oncology-Hematology, Department of Internal Medicine, 986840 Nebraska Medical \\ Center, Omaha 68198-6840, NE, USA. Email: aganti@unmc.edu. \\ Provenance and Peer Review: This article was commissioned by the editorial office, Chinese Clinical Oncology. The article did not undergo external peer review. \\ Comment on: Mok TSK, Wu YL, Kudaba I, et al. Pembrolizumab versus chemotherapy for previously untreated, PD-L1-expressing, locally advanced \\ or metastatic non-small-cell lung cancer (KEYNOTE-042): a randomised, open-label, controlled, phase 3 trial. Lancet 2019;393:1819-30.
}

Submitted Dec 16, 2019. Accepted for publication Jan 09, 2020.

doi: $10.21037 /$ cco.2020.01.05

View this article at: http://dx.doi.org/10.21037/cco.2020.01.05

The management of patients with advanced non-small cell lung cancer (NSCLC), who do not have a targetable driver mutation has come a long way in the past few years, since the advent of immune checkpoint inhibitors. Gone are the days when we used to pick one platinum agent, combine it with a non-platinum agent and hope that the patient in question was one of the lucky $20 \%$ to have a response. The KEYNOTE-024 study demonstrated the superiority of pembrolizumab alone in patients with high PD-L1 expression [Tumor Proportion Score (TPS) $\geq 50 \%$ ] (22C3 antibody) (1). The KEYNOTE-189 and KEYNOTE-407 studies showed the superiority of pembrolizumab in combination with a platinum-based doublet chemotherapy compared to chemotherapy alone in non-squamous NSCLC and squamous cell lung cancer, respectively, with any level of PD-L1 expression (2,3). Similarly, the IMpower 150 study that evaluated carboplatin-paclitaxel-bevacizumab +/- atezolizumab in patients with advanced non-squamous NSCLC found that, similar to KEYNOTE-189, patients who received atezolizumab had a better median overall survival compared to chemotherapy alone (4). Based on these trials, patients who had a high PD-L1 expression receive pembrolizumab alone, while patients with NSCLC with lower expression can receive either pembrolizumab or atezolizumab along with the histology-appropriate, platinum-based chemotherapy.

The results of KEYNOTE-042 should be examined in this context. KEYNOTE-042 was a randomized, open-label, global phase 3 study in adults with treatment naïve, locally advanced or metastatic NSCLC without a sensitizing EGFR mutation or ALK translocation, and a good performance status (ECOG 0-1) and a PD-L1 TPS of $1 \%$ or greater (5). Enrolled patients were randomly assigned to receive pembrolizumab, or investigator's choice of platinum-based chemotherapy for four to six cycles. Overall survival was significantly longer in the pembrolizumab group than in the chemotherapy group in all three TPS populations analyzed. The hazard ratios for survival in the $\geq 50 \%, \geq 20 \%$ and the $\geq 1 \%$ cohorts were 0.69 , (95\% CI, 0.56-0.85); 0.77, (95\% CI, 0.64-0.92) and 0.81 (95\% CI, 0.71-0.93) respectively, favoring pembrolizumab. The median survival in these cohorts for pembrolizumab and chemotherapy were 20.0 vs. 12.2 months, 17.7 vs. 13.0 months, and 16.7 vs. 12.1 months, respectively. Consistent with previous studies, the adverse event profile was better in the pembrolizumab group. There were no new safety signals identified in this study.

These results are in contrast to those seen in the CheckMate 026 trial, that looked at nivolumab in a similar setting (6). In this study, there was no improvement in either progression free survival or overall survival with nivolumab compared to platinum-based chemotherapy in patients with a PD-L1 level (Dako $28-8$ antibody) of $\geq 1 \%$. To add another twist to this story, the recently published results of the CheckMate 227 trial showed that a combination of nivolumab and the CTLA-4 inhibitor, ipilimumab, resulted in a longer overall survival than chemotherapy, independent of the level of PD-L1 expression, as measured by the Dako 28-8 assay (7). Similar to the KEYNOTE-042 study, patients with PD-L1 expression of $\geq 1 \%$ had a median 
overall survival of 17.1 months with the combination immunotherapy, compared to 14.9 months with platinum based chemotherapy. In this study, patients who were treated with nivolumab alone had a median survival of 15.7 months that was similar to the combination of nivolumab and ipilimumab (hazard ratio for the combination 0.90; $95 \%$ CI, 0.76-1.07). More intriguingly, in patients with a PDL1 expression of $<1 \%$, the combination immunotherapy arm had a median overall survival of 17.2 months compared to 12.2 months with chemotherapy. It is unlikely that these discrepancies in the KEYNOTE-042 and the CheckMate 026 and 227 trials are a related to a difference in the methodology used to test for PD-L1 as the 2 assays used namely, $22 \mathrm{C} 3$ and $28-8$ have been shown to be concordant in previous studies (8).

These results raise the important question about the role of cytotoxic chemotherapy in patients whose tumors have a PD-L1 TPS of $\geq 1 \%$. While the KEYNOTE- 042 studied three different cohorts of patients with PDL1 TPS of $\geq 1 \%, \geq 20 \%$ and $\geq 50 \%$, unfortunately they did not report the results of patients with TPS $\geq 1-49 \%$ separately. Given the increasing hazard ratios with inclusion of lower levels of PD-L1 expression in the analysis, it is likely that the majority of the benefit seen in the $\geq 1 \%$ and $\geq 20 \%$ cohorts was driven by the benefit in patients with TPS $\geq 50 \%$. If this is true, then patients with PD-L1 expression of $1-49 \%$ should receive chemoimmunotherapy rather than immunotherapy alone. Thus, results of the KEYNOTE-189, IMPower150 and KEYNOTE-407 studies should guide the management of these patients, who should then be offered a platinum doublet in combination with pembrolizumab. The other option would be to use the results of the CheckMate 227 study and treat these patients regardless of $\mathrm{PD}-\mathrm{L} 1$ expression with a combination of nivolumab and ipilimumab.

Based on the results of the KEYNOTE-042 study, it would be tempting to treat patients were unlikely to tolerate cytotoxic chemotherapy with pembrolizumab alone. However, it must be noted that this study did not include patients with a performance status of ECOG2 and was limited to good performance status patients. Prior studies in PS2 patients have shown that lung cancer patients with a poor performance status, irrespective of age, have an increased incidence of adverse effects with standard chemotherapy and have a poorer overall survival $(9,10)$. Outcomes data with platinum based combination chemotherapy suggest that while the doublet therapy provided superior outcomes compared to monotherapy, these were inferior to those seen in good performance status patients. It is noteworthy that this improvement in survival was at the cost of greater toxicity $(9,11,12)$. If this argument is extended to the current context, it is likely that these patients will tolerate pembrolizumab better and therefore reap the benefits of this drug as well.

In conclusion, the results of the KEYNOTE-042 trial provide another option for the management of patients with advanced NSCLC. However, it is still unclear if patients with low PD-L1 expression have an added benefit from cytotoxic chemotherapy. Our proposed approach would be to use a combination of platinum doublet with pembrolizumab or a combination of nivolumab and ipilimumab in patients who are felt to be able to tolerate these agents. In patients who are not candidates for cytotoxic chemotherapy, options would include a combination of nivolumab and ipilimumab or single agent pembrolizumab, although it would be good to have data specific to these patients. The results of ongoing studies should help answer some of these questions.

\section{Acknowledgments}

Funding: None.

\section{Footnote}

Conflicts of Interest: Both authors have completed the ICMJE uniform disclosure form (available at http://dx.doi. org/10.21037/cco.2020.01.05). Dr. Ganti reports nonfinancial support from Takeda, grants from Apexigen, grants and personal fees from AstraZeneca, personal fees from Roche, grants from Novartis, grants from Merck, outside the submitted work. The other author has no conflicts of interest to declare.

Ethical Statement: The authors are accountable for all aspects of the work in ensuring that questions related to the accuracy or integrity of any part of the work are appropriately investigated and resolved.

Open Access Statement: This is an Open Access article distributed in accordance with the Creative Commons Attribution-NonCommercial-NoDerivs 4.0 International License (CC BY-NC-ND 4.0), which permits the noncommercial replication and distribution of the article with 
the strict proviso that no changes or edits are made and the original work is properly cited (including links to both the formal publication through the relevant DOI and the license). See: https://creativecommons.org/licenses/by-nc-nd/4.0/.

\section{References}

1. Reck M, Rodriguez-Abreu D, Robinson AG, et al. Pembrolizumab versus Chemotherapy for PD-L1Positive Non-Small-Cell Lung Cancer. N Engl J Med 2016;375:1823-33.

2. Gandhi L, Rodriguez-Abreu D, Gadgeel S, et al. Pembrolizumab plus Chemotherapy in Metastatic NonSmall-Cell Lung Cancer. N Engl J Med 2018;378:2078-92.

3. Paz-Ares L, Luft A, Vicente D, et al. Pembrolizumab plus Chemotherapy for Squamous Non-Small-Cell Lung Cancer. N Engl J Med 2018;379:2040-51.

4. Socinski MA, Jotte RM, Cappuzzo F, et al. Atezolizumab for First-Line Treatment of Metastatic Nonsquamous NSCLC. N Engl J Med 2018;378:2288-301.

5. Mok TSK, Wu YL, Kudaba I, et al. Pembrolizumab versus chemotherapy for previously untreated, PD-L1expressing, locally advanced or metastatic non-small-cell lung cancer (KEYNOTE-042): a randomised, open-label, controlled, phase 3 trial. Lancet 2019;393:1819-30.

6. Carbone DP, Reck M, Paz-Ares L, et al. First-Line Nivolumab in Stage IV or Recurrent Non-Small-Cell Lung Cancer. N Engl J Med 2017;376:2415-26.

Cite this article as: Buddharaju LN, Ganti AK. Immunotherapy in lung cancer: the chemotherapy conundrum. Chin Clin Oncol 2020;9(4):59. doi: 10.21037/cco.2020.01.05
7. Hellmann MD, Paz-Ares L, Bernabe Caro R, et al. Nivolumab plus Ipilimumab in Advanced Non-Small-Cell Lung Cancer. N Engl J Med 2019;381:2020-31.

8. Tsao MS, Kerr KM, Kockx M, et al. PD-L1 Immunohistochemistry Comparability Study in Real-Life Clinical Samples: Results of Blueprint Phase 2 Project. J Thorac Oncol 2018;13:1302-11.

9. Sweeney CJ, Zhu J, Sandler AB, et al. Outcome of patients with a performance status of 2 in Eastern Cooperative Oncology Group Study E1594: a Phase II trial in patients with metastatic nonsmall cell lung carcinoma. Cancer 2001;92:2639-47.

10. Ruckdeschel JC, Finkelstein DM, Ettinger DS, et al. A randomized trial of the four most active regimens for metastatic non-small-cell lung cancer. J Clin Oncol 1986;4:14-22.

11. Gronberg BH, Bremnes RM, Flotten O, et al. Phase III study by the Norwegian lung cancer study group: pemetrexed plus carboplatin compared with gemcitabine plus carboplatin as first-line chemotherapy in advanced non-small-cell lung cancer. J Clin Oncol 2009;27:3217-24.

12. Zukin M, Barrios CH, Rodrigues Pereira J, et al. Randomized Phase III Trial of Single-Agent Pemetrexed Versus Carboplatin and Pemetrexed in Patients With Advanced Non-Small-Cell Lung Cancer and Eastern Cooperative Oncology Group Performance Status of 2. J Clin Oncol 2013;31:2849-53. 